\title{
Strain Effect Analysis on Thermal Conductivity of Ge Thin Films
}

\author{
Zhang Xingli $^{1}$, $\quad$ Gong Cuizhi $^{1}$, Wu Guoqiang ${ }^{2}$ \\ ${ }^{1}$ Northeast Forestry University, Harbin150040, China; ${ }^{2}$ The State Key Laboratory of Structural Analysis for Industrial Equipment, Dalian \\ University of Technology, Dalian 116024, China
}

\begin{abstract}
The effects of strains on the thermal conductivity of Ge thin films were studied by nonequilibrium molecular dynamics (NEMD) simulations. The results show that the strains have an appreciable influence on the thermal conductivity of Ge thin films. The thermal conductivity decreases as the tensile strain increases and increases as the compressive strain increases, because of the decrease in the phonons velocities and the surface reconstructions. Meanwhile, a theoretical calculation based on Modified-Callaway model under different strains was performed to verify our NEMD simulation results. We find that the theoretical results closely match the molecular dynamics results. The theoretical analysis reveals that the contributions of the relaxation time on strains are very important to the thermal conductivity of Ge thin films.
\end{abstract}

Key words: thermal conductivity; strains; Ge thin films; molecular dynamics

Ge thin films have attracted strong interest in the scientific community due to their potential applications in thin-film transistors, highly efficient solar cells, and three-dimensional on-chip optical interconnects ${ }^{[1,2]}$. Understanding and predicting thermal transport of Ge thin films at nanoscale are essential for improving the performance and reliability of these devices. Since the characterized size is down to nanoscale, the heat transfer of Ge thin films becomes unusual as compared to that of bulk materials. There are several studies to analyze the effects of the grain sizes and structure defects on the thermal properties of the Ge thin films ${ }^{[3-5]}$. In these studies, the strains are intrinsic and not externally varied for a systematic study. However, nanoscale devices, particularly thin films, usually contain residual strain after fabrication ${ }^{[6]}$, which may considerably affect the thermal transport properties of the material. Many researchers have attempted to simulate the effect of strain on the thermal properties of nanoscale material using different methods. Bhowmicka et al. derived the thermal conductivity as a function of temperature and strain using the Peierls-Boltzmann formulation $^{[7]}$. Zhang et al. investigated how the thermal conductivity responded to the external tensile strain by non-equilibrium molecular dynamics (NEMD) simulations on functionalized bilayer graphene sheet ${ }^{[8]}$. Wang et al. investigated the thermal properties of $\mathrm{Ni} / \mathrm{Al}$ laminated structures using NEMD method ${ }^{[9]}$. Xv et al. presented a model that combined lattice dynamics and the phonon Boltzmann transport equation (BTE) to analyze strain effect on the cross-plane phonon thermal conductivity of silicon wire-germanium host nanocomposites ${ }^{[10]}$. While all these studies have shown the significance of strain on the nanoscale thermal transport, few researches have been conducted on the Ge thin films.

In the present work, we carried out NEMD simulations and theoretical calculations to investigate the effects of strains on the thermal properties of $\mathrm{Ge}$ thin films. By comparing the predictions of the two methods, we analyzed the physical mechanisms of the tensile and compressive strain effect on the thermal conductivity of the thin films in detail.

$\overline{\text { Received date: February 02, }} 2016$

Foundation item: Fundamental Research Funds for the Central Universities of China (DL12BB38)

Corresponding author: Zhang Xingli, Ph. D., College of Mechanical and Electrical Engineering, Northeast Forestry University, Harbin150040, P. R. China, Tel: 0086-451-82192357, E-mail: cindy07b@126.com

Copyright (C) 2017, Northwest Institute for Nonferrous Metal Research. Published by Elsevier BV. All rights reserved. 


\section{Molecular Dynamic Simulation}

The NEMD simulations of the Ge thin films are performed using LAMMPS code. Fig.1 shows the simulation model in the present research. At the initialized condition, the positions of each atom are arranged according to the crystal lattice structure, and atomic velocities meet the Maxwell-Boltzmann distribution function. The sizes of the simulation cells are $10 A_{0}, 4 A_{0}$ and $4 A_{0}$ in the $X, Y$ and $Z$ directions, respectively, where $A_{0}$ is the lattice constant of Ge. The heating and cooling zones with the thickness of $3 A_{0}$ create a temperature gradient in the system by controlling the energy given or taken from these regions. Adiabatic zones with the thickness of $2 A_{0}$ are constructed to prevent the atoms escaping from the system, and the velocities of each atom in this region are 0 .

Tersoff three-body potential model is employed to describe the interaction among different $\mathrm{Ge}$ atoms which is reliable to describe the semiconductor atoms potential properties $^{[11]}$. In our simulations, the Newton's classical equations of motion for those atoms are solved with the velocity-Verlet integration algorithm. The time step for the simulation is set as $1 \mathrm{fs}$. The simulations consist of two stages: the first stage is the constant-temperature simulation, in which the temperature is maintained at constant value with a coupling time of $2 \times 10^{6} \mathrm{MD}$ steps; the second stage is the constant-energy one with a coupling time of $8 \times 10^{6} \mathrm{MD}$ steps to ensure the whole system reaching an equilibrium state. The method proposed by Jund ${ }^{[12]}$ is used to apply a specified heat flux by scaling the velocities of the atoms in the heating and cooling zones of the simulations. A specified amount of energy $\Delta E$ (set to $1 \%$ of $K_{\mathrm{B}} T$, ) is added to heating zone and the same amount is subtracted from the cooling zone at each time step.

The velocities $v_{i}$ of all the particles in the heating and cooling zones are scaled according to the following equation:

$v_{i, \text { new }}=v_{\mathrm{G}}+\alpha\left(v_{i, \text { old }^{-}} v_{\mathrm{G}}\right)$

Where, $v_{\mathrm{G}}$ is the velocity of the center of mass, which is set as zero at the beginning of our simulations. A scaling factor $\alpha$ is calculated from the following equation:

$$
\alpha=\sqrt{1 \pm \frac{\Delta E}{E_{k}}}
$$

Where, $E_{k}=\sum_{i=1}^{N} \frac{1}{2} m_{i} v_{i}^{2}$ is the kinetic energy of atoms in the heating and cooling zones.

Based on the Fourier law of conduction, the thermal conductivity is given as:

$$
\lambda=\frac{\Delta E}{2 A \tau \cdot(\partial T / \partial x)}
$$

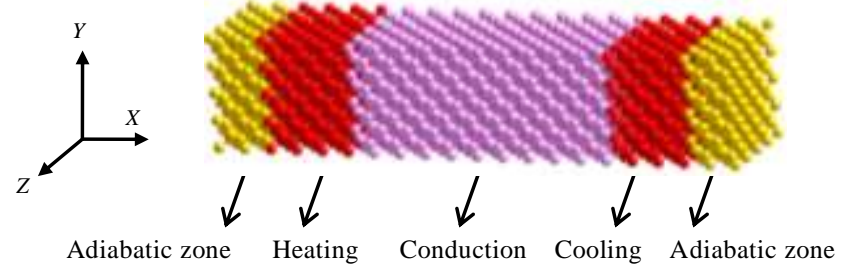

Fig.1 MD simulation model in Ge thin film

Where, $\tau$ is the simulation step time; $A$ is the cross-sectional area. To obtain the temperature distribution, the system is divided into 10 planes along the longitudinal direction, and the instantaneous temperature of each plane is calculated using the following equation:

$$
T=\frac{1}{3 K_{\mathrm{B}} N}\left\langle\sum_{i=1}^{N}\left(\frac{1}{2} m_{i} v_{i}^{2}\right)\right\rangle
$$

Where, $m_{i}$ is the mass of atoms; $v_{i}$ is the velocity of atoms; $N$ is the number of atoms in each plane; $K_{\mathrm{B}}$ is Boltzmann constant.

For the analyses, $0 \% \sim 15 \%$ tensile and compressive strains are imposed on our NEMD simulation system by recalibrating the atomic coordinates. The two outermost atomic planes of the thermal conducting zones at the $X$ direction are selected to be applied displacement load with an opposite direction. After each load applied process is finished, the system needs to reach equilibrium for a certain relaxation time. In our simulations, the rate of applying displacement load is $0.005 \mathrm{~nm}$ per $10^{5}$ steps.

\section{Results and Discussion}

\subsection{NEMD predictions}

The temperatures of the NEMD simulations are 300, 500, $700 \mathrm{~K}$. As the film strain is changed in the range of $-0.15 \sim 0.15$, the thermal conductivities of Ge thin films are collected in Fig.2. As shown in the figure, the presence of strains has great impact on thermal transport for $\mathrm{Ge}$ thin films, and the thermal conductivity dependence of strains becomes less sensitive as temperature increases. The thermal conductivity decreases as the tensile strain increases and increases as the compressive strains increases. When the tensile strain reaches 0.15 , the thermal conductivity of Ge thin films at $500 \mathrm{~K}$ decays drastically by $31 \%$; and when the compressive strain is equal to 0.15 , the relative thermal conductivity increases by about $17 \%$. Such trend is consistent with the earlier reported analyses of other nanostructures in Ref.[13, 14].

To explain the coupling between strains and thermal conductivity, we calculate the phonon density of states (PDOS) for different strains which can describe accurately the thermal transport property of the material. Fig.3 shows that the peaks of PDOS almost coincide for different strains 


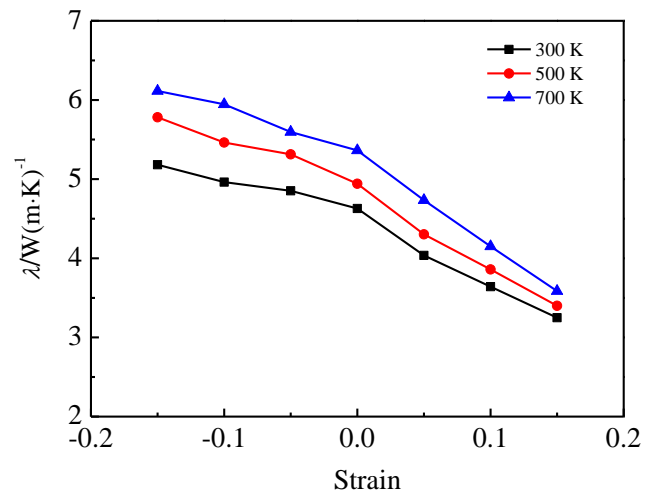

Fig.2 Thermal conductivity variation with strains

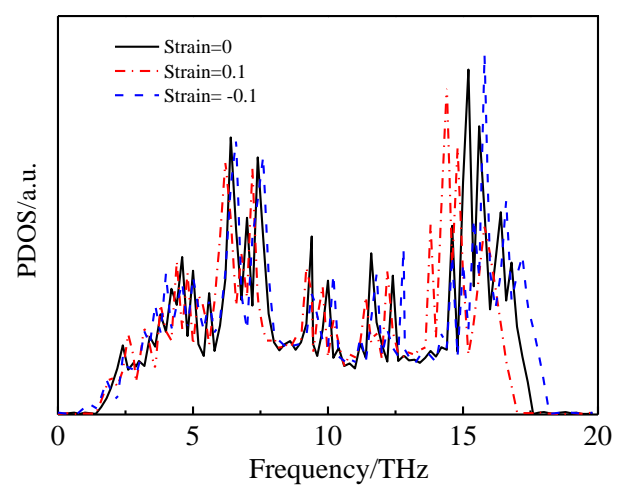

Fig.3 Strain dependent phonon density of state of Ge thin films

at low frequencies, and they are more sensitive to the strains at high frequencies. Meanwhile the peaks move to the left when the tensile strain is applied, while they move to the right for the compressive strain. The results imply that the tensile strains can decline peaks of PDOS remarkably at high frequencies, which could slow down the phonon group velocities. However, the compressive strains could enhance thermal transportation capacity by making the phonons carry more energy at high frequencies ${ }^{[15]}$.

In gas kinetic theory, the lattice thermal conductivity $\lambda$ can be calculated using the following expression:

$$
\lambda=\frac{1}{3} C v \Lambda_{\text {eff }}
$$

Where, $C$ is the heat capacity per unit volume; $v$ and $\Lambda_{\text {eff }}$ are the velocity of sound and mean free path of the phonons, respectively. It can be seen from Eq.(5) that the decline of phonon velocities due to the tensile strains will decrease the thermal conductivity of thin films.

On the other hand, the surface reconstructions of the simulation system contribute to the change in thermal conductivity. In Fig.4, the Ge thin films under different tensile strains exhibit different atomic configuration
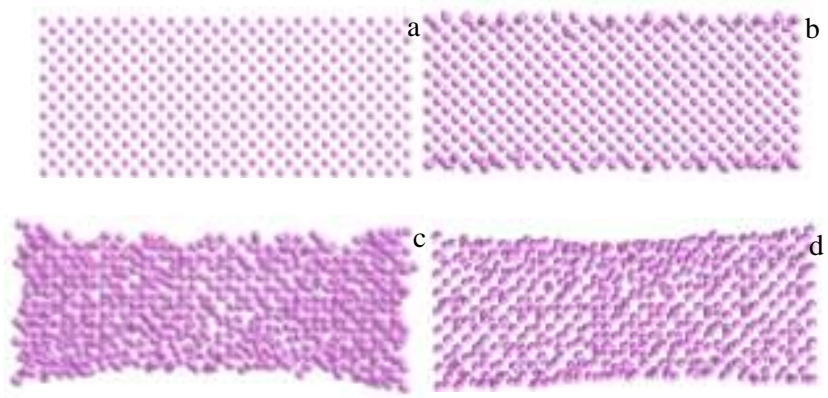

Fig.4 Configuration plots of Ge thin films as the tensile strain increases: (a) $0 \%$, (b) $5 \%$, (c) $10 \%$, and (d) $15 \%$

characteristics. Under small strain (Fig.4a and 4b), the surface disorder is significantly small, and there is no plastic deformation in the simulation system. With the increase of strain, the atoms recombine with each other to form new bonds, thus the disordered region expands to the whole conducting zones and the plastic deformation occurs gradually. Surface reconstruction is one of the main factors that can greatly reduce the thermal conductivity of the thin films. It may be due to that the tensile strain brings about increasing atomic potential energy vibrations of the thin films ${ }^{[16]}$.

\subsection{Theoretical approach}

We also performed a theoretical analysis on the Boltzmann transport equation (BTE) to verify our NEMD simulation results. The phenomenological model developed by Callaway is a useful approach to predict the thermal conductivity of bulk solids, but the difference between longitudinal waves and transverse waves of the phonons has not been taken into account in this method ${ }^{[17]}$. Palmer has proposed a Modified-Callaway model which could calculate the phonons relaxation time more exactly ${ }^{[18]}$. According the Modified-Callaway theory, the lattice thermal conductivities consist of one longitudinal wave thermal conductivity and two transverse wave thermal conductivities, so the thermal conductivity can be expressed as:

$$
\begin{aligned}
& \lambda=\lambda_{\mathrm{L}}+2 \lambda_{\mathrm{T}} \\
& \lambda_{\mathrm{T}}=\lambda_{\mathrm{T} 1}+\lambda_{\mathrm{T} 2} \\
& \lambda_{\mathrm{T} 1} \text { and } \lambda_{\mathrm{T} 2} \text { can be described as: } \\
& \lambda_{\mathrm{T} 1}=\frac{1}{3} C_{\mathrm{T}} T^{3} \int_{0}^{\theta / T} \frac{\tau_{\mathrm{C}}^{\mathrm{T}} x^{4} \mathrm{e}^{x}}{\left(\mathrm{e}^{x}-1\right)^{2}} \mathrm{~d} x \\
& \lambda_{\mathrm{T} 2}=\frac{1}{3} C_{\mathrm{T}} T^{3} \frac{\left[\int_{0}^{\theta / T} \frac{\tau_{\mathrm{C}}^{\mathrm{T}} x^{4} \mathrm{e}^{x}}{\tau_{\mathrm{N}}^{\mathrm{T}}\left(\mathrm{e}^{x}-1\right)^{2}} \mathrm{~d} x\right]}{\int_{0}^{\theta / T} \frac{\tau_{\mathrm{C}}^{\mathrm{T}} x^{4} \mathrm{e}^{x}}{\tau_{\mathrm{N}}^{\mathrm{T}} \tau_{\mathrm{R}}^{\mathrm{T}}\left(\mathrm{e}^{x}-1\right)^{2}} \mathrm{~d} x}
\end{aligned}
$$

Generally, the reduction in the lattice thermal conductivity 
can be realized by employing the following major processes: the phonon Umklapp scattering, normal three-phonon scattering, boundary scattering, and point-defect scattering. So the phonon scattering relaxation rate $\tau_{\mathrm{C}}{ }^{-1}$ is equal to:

$$
\tau_{\mathrm{C}}^{-1}=\tau_{\mathrm{U}}^{-1}+\tau_{\mathrm{N}}^{-1}+\tau_{\mathrm{B}}^{-1}+\tau_{\mathrm{D}}^{-1}
$$

The relaxation time of Umklapp scattering processes is defined as ${ }^{[19]}$ :

$$
\begin{aligned}
& \left(\tau_{\mathrm{U}}^{-1}\right)_{i}=B_{\mathrm{U}} \omega^{2} T^{3} \mathrm{e}^{-\theta_{i} / 3 T} \\
& \boldsymbol{B}_{\mathrm{U}} \approx \frac{\hbar \gamma_{i}^{2}}{\boldsymbol{M} v_{i} \theta_{i}}
\end{aligned}
$$

Where, $\hbar$ is Planck constant; $\theta_{i}$ is the Debye temperature, which is $374 \mathrm{~K}$ for $\mathrm{Ge}$; $v_{i}$ is the speed of sound; $M$ is the average mass of all atoms.

The relaxation time of three-phonon scattering can be written as ${ }^{[20]}$ :

$$
\begin{aligned}
& \left(\tau_{\mathrm{N}}^{-1}\right)_{\mathrm{T}}=B_{\mathrm{N}}^{\mathrm{T}} \omega T^{4},\left(\tau_{\mathrm{N}}^{-1}\right)_{\mathrm{L}}=B_{\mathrm{N}}^{\mathrm{T}} \omega^{2} T^{3} \\
& B_{\mathrm{N}}^{\mathrm{T}} \approx \frac{k_{\mathrm{B}}^{4} \gamma_{\mathrm{T}}^{2} V_{0}}{M h^{3} v_{i}^{5}}, B_{\mathrm{N}}^{\mathrm{L}} \approx \frac{k_{\mathrm{B}}^{3} \gamma_{\mathrm{L}}^{2} V_{0}}{M h^{2} v_{i}^{5}}
\end{aligned}
$$

The relaxation time due to the boundary scattering depends on the phonon group velocity, so it can be expressed as:

$$
\tau_{\mathrm{B}}^{-1}=v_{i} / L
$$

Where, $L$ is the characteristic grain size.

The relaxation time due to point-defect scattering is supposed as 0 . We introduce the dilation of volume to represent the strain $\varepsilon, \varepsilon$ is equal to:

$$
\varepsilon=\frac{V}{V_{0}}
$$

Where, $V$ is the atomic volume when the superlattices materials are applied strains; $V_{0}$ is the atomic volume. Clearly, $\varepsilon<1$ implies compressive strain, while $\varepsilon>1$ implies tensile strain. The phonon frequencies are expected to scale with strain as ${ }^{[7]}$ :

$$
\omega=\omega_{0} \varepsilon^{-\gamma_{i}}
$$

Where, $\omega_{0}$ is the Debye frequency; $\gamma_{i}$ is Grüneisen parameter.

Fig.5 shows the comparison of thermal conductivities of Ge thin films calculated from the NEMD and Modified-Callaway theory for various levels of strains. From Fig.5 it can be seen that they are in excellent agreement with each other at the temperature of $500 \mathrm{~K}$. Our theoretical analysis shows the strain dependence of the thermal conductivity arises from the relaxation time on strain, and this result is consistent with the formulation of Peierls ${ }^{[21]}$. According to Peierls, thermal conductivity has been shown as $\lambda=\sum \tau(\mathrm{k}) C(\mathrm{k}) v(\mathrm{k}) \nu(\mathrm{k})$, where $C$ is the heat capacity of the mode. It is indicated that the strain dependence of thermal conductivity is determined entirely by the strain dependence of the group velocity $v$, and the relaxation time $\tau$.

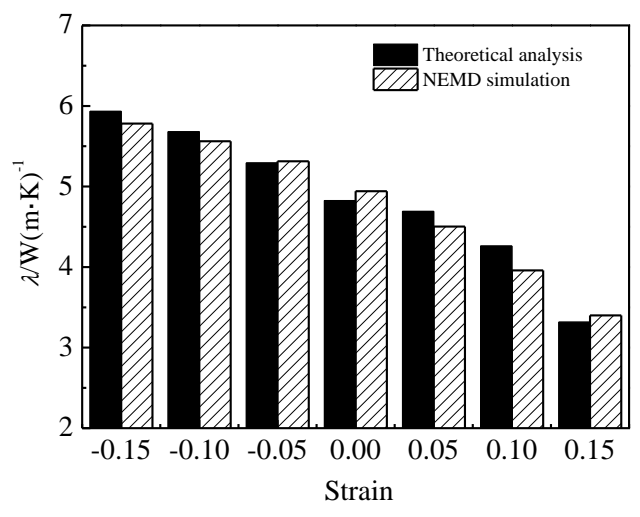

Fig.5 Computation comparison of NEMD and theoretical model at different strains

\section{Conclusions}

1) The NEMD approach has been primarily used for simulating the dependence relations between thermal conductivity and strains of Ge thin films.

2) The thermal conductivity is strongly affected by tensile strains and compressive strains at different temperatures. The thermal conductivity decreases as the tensile strain increases and increases as the compressive strains increases. Generally, the variation of thermal conductivity in thin films imposed on strains is believed to be caused by the decrease of the phonons velocities and surface reconstructions generated under strains.

3) We have also introduced the Modified-Callaway theory to assess the accuracy of the NEMD-predicted values. Thermal properties calculated from the two methods lead to quite a coincident results.

\section{References}

1 Sadoh T, Kamizuru H, Kenjo A et al. Applied Physics Letters[J], 2006, 89: 192114

2 Michel J, Liu J, Kimerling L C. Nature Photon[J], 2010, 4: 527

3 Wang J, Kulkarni A J, Ke F J et al. Computer Methods in Applied Mechanics and Engineering[J], 2008, 197: 3182

4 Zhang X L, Wu G Q. Rare Metal Materials and Engineering [J], 2015, 44(3): 656 (in Chinese)

5 Zhang X L, Sun Z W. Rare Metals[J], 2011, 30(4): 317

6 Adamcyk M, Schmid J H, Tiedje T. Applied Physics Letters[J], 2002, 80: 4357

7 Bhowmick S, Shenoy V B. The Journal of Chemical Physics [J], 2006, 125: 164513

8 Zhang Y Y, Pei Q X, He X Q et al. Chemical Physics Letters[J], 2015, 622: 104

9 Wang Xin, Shen Shengping. Computational Materials Science [J], 2014, 84: 13 
10 Xu Y, Lia G. Journal of Applied Physics[J], 2009, 106: 114 302

11 Tersoff J. Physical Review B[J], 1988, 37: 6991

12 Jund P, Jullien R. Physical Review B[J], 1999, 59: 13707

13 Kwangsub J, Maenghyo C, Min Z. Journal of Applied Physics[J], 2012, 112: 083522

14 Xiaobo Li, Kurt M, Martin L et al. Physical Review B[J], 2010, 81: 245318

15 Xu Z P, Buehler M J. Nanotechnology[J], 2009, 20: 185701

16

Zhang Jianwei, He Xiaodong, Yang Lin et al. Sensors[J],
2013, 13: 9388

17 Callaway J. Physical Review[J], 1959, 113: 1046

18 Palmer A, Bartkowski K, Gmelin E. Physical Review B $[\mathrm{J}]$, 1997, 56: 9431

19 Morelli D, Heremans J, Slack G. Physical Review B[J], 2002, 66: 195304

20 Herring C. Physical Review[J], 1954, 95: 954

21 Peierls R E. Quantum Theory of Solids[M]. Oxford: Oxford University Press, 1956: 106

\title{
应变影响下单晶 $\mathrm{Ge}$ 薄膜热导率分析
}

\author{
张兴丽 ${ }^{1}$, 巩翠芝 ${ }^{1}$, 吴国强 ${ }^{2}$ \\ (1. 东北林业大学, 黑龙江 哈尔滨 150040) \\ (2. 大连理工大学 工业装备结构分析国家重点实验室, 辽宁 大连 116024)
}

\begin{abstract}
摘 要: 利用非平衡态分子动力学模拟方法研究了应变对Ge薄膜热导率的影响。结果表明系统应变对单晶Ge薄膜热导率产生明显影响, 热导率随着拉伸应变的增大而减小, 随着压缩应变的增大而增大, 得出声子速率降低以及薄膜表面重构是产生该模拟结果的内在原因。 同时，采用修正的Callaway模型对NEMD结果进行理论验证，2种方法得到的结果吻合得较好。理论结果表明应变驰豫时间对Ge单晶薄 膜的热导率产生了重要影响。
\end{abstract}

关键词: 热导率; 应变; $\mathrm{Ge}$ 薄膜; 分子动力学

作者简介: 张兴丽, 女, 1981 年生, 博士, 讲师, 东北林业大学机电工程学院, 黑龙江 哈尔滨 150040, 电话: 0451-82192851, E-mail: zhang-xingli@nefu.edu.cn 